\title{
Some open questions concerning the modelling of non-locality in Reynolds stress type models of stellar convection.
}

\author{
F. Kupka ${ }^{1}$ \\ ${ }^{1}$ Max-Planck-Institute for Astrophysics, Karl-Schwarzschild-Str. 1, 85741 Garching, Germany \\ email: fk@mpa-garching.mpg.de
}

\begin{abstract}
We discuss some benefits and pitfalls when combining conceptually different types of closure approximations into complete Reynolds stress models of stellar convection.
\end{abstract}

Keywords. Convection, turbulence, stars: interiors

\section{Introduction and motivation}

Combining different concepts for closure approximations in non-local Reynolds stress models of convection may appear attractive for improving their overall performance. Here, we provide an outline of some closure relations and their origin and discuss the consequences for solutions of Reynolds stress models when putting them together.

\section{Closed Reynolds stress models and region of applicability}

Several useful closure relations have been identified during numerical simulation studies presented in this volume. Relevant quantities include: 1) the dissipation rate of temperature fluctuations $\epsilon_{\theta}$ (Canuto 1992, Canuto \& Dubovikov 1998 (CD98); not shown here); 2 ) the flux of the rate of kinetic energy dissipation $\langle\epsilon w\rangle$ (Canuto 1992; see Kupka \& Muthsam 2007c); 3) the third order moments (TOMs) $\left\langle w^{2} \theta\right\rangle$ and $\left\langle w \theta^{2}\right\rangle$ (Gryanik \& Hartmann 2002 (GH 2002) and Mironov et al. 1999, with $\left\langle w^{2} \theta\right\rangle$ also suggested by CD98; see Kupka \& Robinson 2007 and Kupka \& Muthsam 2007b); 4) the fourth order moments of vertical velocity and temperature (GH 2002 and Gryanik et al. 2005; see Kupka \& Robinson 2007); 5) the pressure flux $\left\langle p^{\prime} w\right\rangle$ (Canuto 1997, see Kupka \& Muthsam 2007c). They all have their own region of applicability. The relation for $\epsilon_{\theta}$ has been derived from a model developed primarily for isotropic turbulence. The relation for $\langle\epsilon w\rangle$ has been obtained from dimensional scaling and eddy damping arguments. The relations for $\left\langle w^{2} \theta\right\rangle,\left\langle w \theta^{2}\right\rangle$, and for fourth order moments have been obtained from scaling arguments applied to mass-flux averages. The relation for $\left\langle p^{\prime} w\right\rangle$ is based on a polytrope model for relations between fluctuations of pressure, density, and temperature.

The models for $\langle\epsilon w\rangle$ and $\left\langle w \theta^{2}\right\rangle$ work well for a large physical parameter space in both stable and unstable stratification. This essentially holds for $\epsilon_{\theta}$ and $\left\langle w^{2} \theta\right\rangle$, too, while the models for fourth order moments are mainly useful for the interior part of convection zones (Kupka \& Robinson 2007). The model for $\left\langle p^{\prime} w\right\rangle$ requires a more complex prescription for the polytropic index $(n=1+1 / m)$ to avoid case dependent parameter adjustments. Putting these ingredients together does not imply an improved performance of the complete model. For example, Kupka \& Robinson (2007) and Kupka \& Muthsam (2007b) show that the relation $\left\langle w \theta^{2}\right\rangle=\left\langle\theta^{3}\right\rangle /\left\langle\theta^{2}\right\rangle\langle w \theta\rangle$ (GH2002) is an excellent approximation for very different types of convection zones. Nevertheless, its application to the 
convective core of a massive star results in a convectively neutral centre (Roxburgh \& Kupka 2007), which does not appear very plausible (similar can happen with less promising closure relations, see Roxburgh \& Kupka 2007). The Reynolds stress model applied in Kupka \& Montgomery (2002) and Montgomery \& Kupka (2004) to envelope convection zones used the relations for $\epsilon_{\theta}$ and $\langle\epsilon w\rangle$ mentioned above. Both are compatible with the overall strategy of Canuto $(1992,1993,1997)$ and CD98 of using damping time scales to describe pressure correlations and dissipation processes. However, this model has problems for efficient convection zones (Kupka \& Muthsam 2007a,b). Could a 'hybrid model' provide better prospects?

\section{An intermediate model for third order moments}

Such a model has been proposed by CD98. Considering the traditional mass-flux approach they suggested to use the mass-flux relations $\left\langle w^{2} \theta\right\rangle=\left\langle w^{3}\right\rangle /\left\langle w^{2}\right\rangle\langle w \theta\rangle$ (cf. GH2002) and $\left\langle w \theta^{2}\right\rangle=\left\langle w^{3}\right\rangle /\left\langle w^{2}\right\rangle\langle w \theta\rangle\left(\left\langle\theta^{2}\right\rangle /\left\langle w^{2}\right\rangle\right)^{1 / 2}$ as approximations for the Reynolds averages. The other TOMs were supposed to be computed with the eddy damped model of Canuto (1992, 1993). Being computationally less expensive than the complete eddy damped model, but more costly than the down-gradient approximation, it was called 'intermediate model'. Solutions of the closed Reynolds stress model using this intermediate model have been calculated with a modified version of the code of Kupka (1999). In Fig. 1 its results are compared with other Reynolds stress models and ensemble averages obtained from 3D numerical simulations with the ASCIC code (Muthsam et al. 1995, 1999) for the case of inefficient, shallow convection (cf. Kupka \& Muthsam 2007a,b). Several properties of this 'CD98 intermediate TOM model' are remarkable. First of all, even with $\left\langle p^{\prime} w\right\rangle=0$ the super-adiabatic temperature gradient remains clearly positive within the interior of the convection zone. The convective flux is comparable to the full model (Kupka \& Montgomery 2002), but convection is found to be less efficient in the interior. The remaining discrepancy clearly calls for including a model of $\left\langle p^{\prime} w\right\rangle$. But despite the scaling relation used for $\left\langle w^{2} \theta\right\rangle$ works very well on its own, the coupled Reynolds stress model is much less successful in recovering this quantity. The cross-correlations do not improve much compared to the full TOM model, but their functional form appears more smooth, as in the simulations. The relation used for $\left\langle w \theta^{2}\right\rangle$ violates a symmetry with respect to sign-change of $\theta$, which explains the wrong sign of the closed model within the overshooting region (this is not the case for the GH2002 model shown in a consistency test for comparison).

\section{Discussion of results and conclusions}

Would results improve, if the GH 2002 expression for $\left\langle w \theta^{2}\right\rangle$ were used in this intermediate model? With some modifications the approach of CD98 has been repeated. The answer is negative: this variant is plagued by instabilities. Evidently, the TOM models need further improvements before offering progress beyond the model used in Kupka \& Montgomery (2002) and Montgomery \& Kupka (2004). The stellar parameter range of the latter may be extended to envelope convection in RR Lyrae stars, perhaps Cepheids, and convection zones driven by Fe peak opacities. Deep, quasi-adiabatic convection zones appear off limits. Further improvements are required to replace down-gradient type nonlocal convection models. In the form suggested by Xiong $(1978,1986)$ and variants thereof this class of models has already been applied to a large variety of stellar convection zones by Prof. Xiong and his co-workers. However, the limitations found in Kupka \& Muthsam (2007a,b,c) corroborate the claim already known in the atmospheric sciences community 

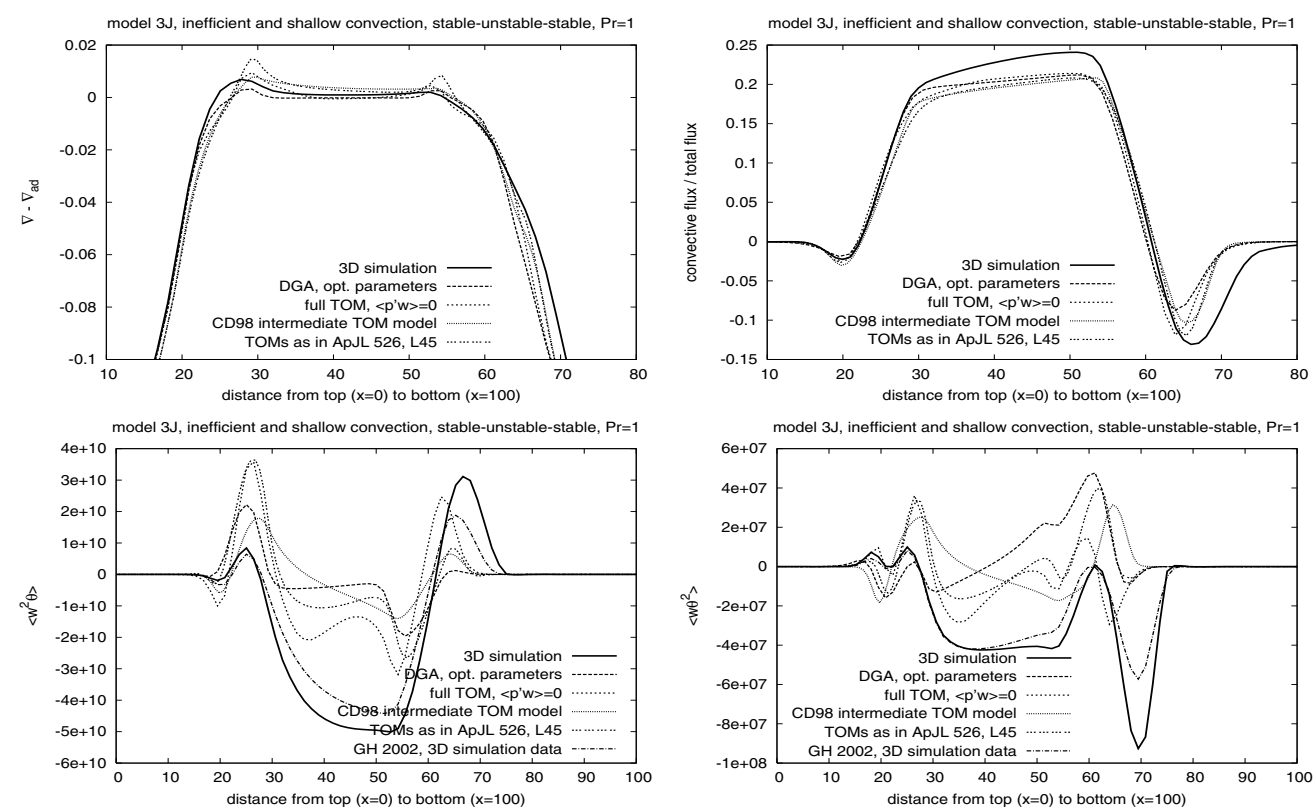

Figure 1. Super-adiabatic temperature gradient, relative convective flux (upper left and right panels) as well as $\left\langle w^{2} \theta\right\rangle$ and $\left\langle w \theta^{2}\right\rangle$ (lower left and right panels) for case '3J'. Simulation data are compared to complete Reynolds stress models. In addition, the GH2002 TOM models are shown with input data taken from the 3D simulations.

(Canuto et al. 1994) that this class of models for the TOMs is incomplete. Improved hybrid models would be appreciated by a large community of users.

\section{References}

Canuto, V. M. 1992, ApJ 392, 218 (C'92)

Canuto, V. M. 1993, ApJ 416, 331 (C'93)

Canuto, V. M. 1997, ApJ 482, 827 (C'97)

Canuto, V. M., Minotti, F., Ronchi, C., Ypma, R. M. \& Zeman, O. 1994, J. Atmos. Sci. 51, 1605

Canuto, V. M. \& Dubovikov, M. S. 1998, ApJ 493, 834 (CD98)

Gryanik, V. M. \& Hartmann, J. 2002, J. Atmos. Sci. 59, 2729

Gryanik, V. M., Hartmann, J., Raasch, S. \& Schröter, M. 2005, J. Atmos. Sci. 62, 2632

Kupka, F. 1999, ApJL 526, L45

Kupka, F. \& Montgomery, M. H. 2002, MNRAS 330, L6 (KM2002)

Kupka, F. \& Muthsam, H. J. 2007a, this volume p. 80

Kupka, F. \& Muthsam, H. J. 2007b, this volume p. 83

Kupka, F. \& Muthsam, H. J. 2007c, this volume p. 86

Kupka, F. \& Robinson, F. J. 2007, this volume p. 74

Mironov, D. V., Gryanik, V. M., Lykossov, V. N. \& Zilitinkevich, S. 1999, J. Atmos. Sci. 56, 3478

Montgomery, M. H. \& Kupka, F. 2004, MNRAS 350, 267

Muthsam, H. J., Göb, W., Kupka, F., Liebich, W. \& Zöchling, J. 1995, Astron. \& Astrophys. 293,127

Muthsam, H. J., Göb, W., Kupka, F. \& Liebich, W. 1999, New Astronomy 4, 405

Roxburgh, I. W. \& Kupka, F. K. 2007, this volume p. 77

Xiong, D. R. 1978, Chinese Astronomy 2, 118

Xiong, D. R. 1986, Astron. \&3 Astrophys. 167, 239 